\title{
Dynamic Structure Factor in Liquid Cesium on The Basis of Time-Scale Invariance of Relaxation Processes ${ }^{\text {II }}$
}

\author{
R. M. Yulmetyev*, A. V. Mokshin*, P. Hänggi**, and V. Yu. Shurygin*** \\ * Kazan State Pedagogical University, 420021 Kazan, Russia \\ email: rmy@dtp.ksu.ras.ru;mav@dtp.ksu.ras.ru \\ ** University of Augsburg, D-86135 Augsburg, Germany \\ *** Yelabuga State Pedagogical Institute, 423630 Yelabuga, Russia \\ Received June 7, 2002
}

\begin{abstract}
The dynamic structure factor $S(k, \omega)$ in liquid cesium near the melting point at $T=308 \mathrm{~K}$ is studied by means of Zwanzig-Mori's memory function formalism. The spectra of $S(k, \omega)$ are calculated on the basis of the idea of time-scale invariance of relaxation processes in liquid metals, which appeared on the fourth relaxation level. The obtained spectra of $S(k, \omega)$ are compared with the results of an inelastic neutron scattering measurement. For the description of memory effects we use the statistical presentation about the non-Markovity parameter $\varepsilon_{1}(k, \omega)$. We find that collective excitations at low wave-vector values have a non-Markovian nature. (C) 2002 MAIK "Nauka/Interperiodica”.
\end{abstract}

PACS numbers: 61J2.-q; 61.20.-p; 67.40.Fd

The study of collective effects in simple liquids has been the subject of intense interest over the last few years in Inelastic Neutron Scattering (INS) experiments [1], Molecular Dynamics (MD) simulations [2,3] and different theories [4]. According to these methods, liquid alkali metals have distinct collective excitations in a wide range of wave-vector values and also outside the hydrodynamic region. This fact was obtained from a careful analysis of the dynamic structure factor $S(k, \omega)$ spectra. High-frequency peaks are appreciable in $S(k$, $\omega)$ for low $k$ values (approximately, up to half the position of the first maximum of the static structure factor), and these peaks do not exist in high- $k$ regions. Understanding the microscopic mechanism responsible for the propagation and the damping of these excitations is still a challenge in liquid metals, where the dynamics is conditioned by the interacting electron gas effects. So, a recent INS experiment on liquid cesium near its melting point has been done by Bodensteiner et al. at Grenoble [5]. The results of their experiment have shown that the high-frequency collective excitations exist in this system for wave vector region $k<1.1 \AA^{-1}$. MD simulation performed by Kambayashi and Kahal [2] validated fully the findings of INS in liquid cesium. The analysis of collective excitations, extending beyond the hydrodynamic limit, is a great contribution to the development of theoretical models of the liquid state [6].

\footnotetext{
II This article was submitted by the authors in English.
}

For a system composed of $N$ particles of mass $m$, the density fluctuations are given by

$$
\rho(k, t)=N^{-1} \sum_{j=1}^{N} \exp \left[i k r_{j}(t)\right],
$$

where $r_{j}(t)$ is the coordinate of the $j$ th particle, and $k$ is the wave vector. Then, the main dynamical quantity of interest is the density-density correlation function $F(k$, $t)=\left\langle\rho^{*}(k, 0) \rho(k, t)\right\rangle\left\langle\left\langle|\rho(k, 0)|^{2}\right\rangle\right.$, where $\left\langle|\rho(k, 0)|^{2}\right\rangle=S(k)$ is the static structure factor. The angular brackets denote an equilibrium ensemble average at temperature $T$ and density $\rho=N / V$ with $V$ being the volume of the system involved. If $F(k, t)$ is known, the dynamic structure factor follows from

$$
S(k, \omega)=[S(k) / \pi] \lim _{\epsilon \rightarrow+0} \operatorname{Re}[F(k, i \omega+\epsilon)] .
$$

According to the memory function (MF) formalism and the projection operator method [7], we can find the time evolution of $F(k, t)$ in the following way:

$$
\frac{d F(k, t)}{d t}=-\Omega_{1}^{2} \int_{0}^{t} d \tau M_{1}(t-\tau) F(k, \tau) .
$$

Here we introduce the first general relaxation frequency parameter $\Omega_{1}^{2}$ and the MF of the first order $M_{1}(k, t)$. However, by using the same method, we can define the time evolution of the high order $M F M_{1}(k, t), M_{2}(k, t)$, ... Thus, a set of interconnected relaxation processes corresponds to an arbitrary relaxation process (for 


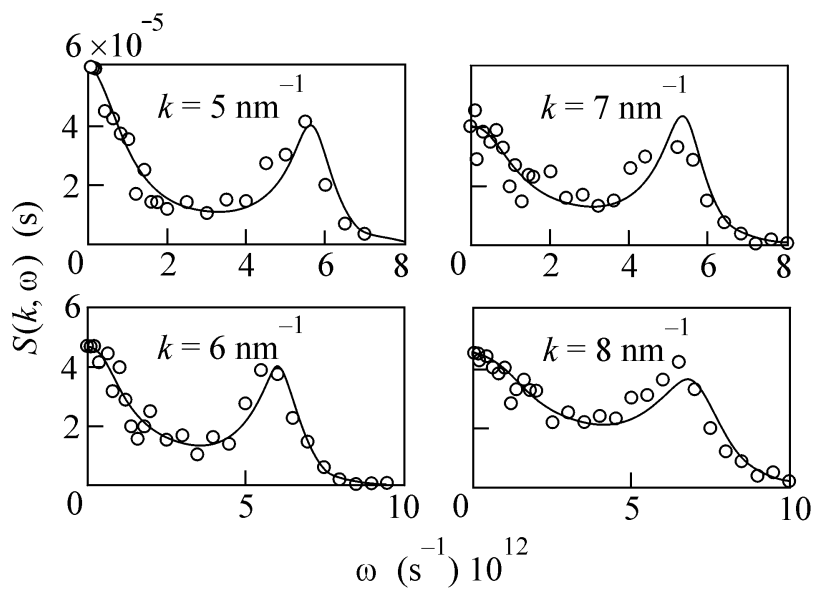

Fig. 1. Theoretical (solid line) and experimental $(O)$ values of dynamic structure factor for liquid cesium near the melting point

example, to a density fluctuations in liquids), which can be easily taken into account by MF formalism.

According to the definition, for ergodic processes the correlation functions $M_{0}(k, t)=F(k, t), M_{1}(k, t), \ldots$, $M_{i}(k, t)$ have the following properties:

$$
\lim _{t \rightarrow 0} M_{i}(k, t)=1, \quad \lim _{t \rightarrow \infty} M_{i}(k, t)=0 .
$$

Thus, the correlation functions $M_{i}(k, t)$ have characteristic time scales, which can generally be defined at fixed $k$ by the equation

$$
\tau_{i}=\operatorname{Re}\left[\tilde{M}_{i}(0)\right], \quad \tilde{M}_{i}(s)=\int_{0}^{\infty} d t M_{i}(t) e^{-s t} .
$$

Here $\operatorname{Re}[\ldots]$ denotes the real part of $[\ldots]$.

These time scales $T_{i}$ characterize the corresponding relaxation processes and can have different numerical values. Nonetheless, on a certain level, (for example, on the $i$ th level) the scale invariance of the nearest interconnected relaxation processes can exist. Physically it implies the existence of the time-scale invariance (TSI) of relaxation processes on the nearest $i$ th and $(i+1)$-th relaxation levels. Such an approach allows one to receive an approximation of the form $M_{i+1}(k, t) \approx$ $M_{i}(k, t)$, which is actually the closure of the chain of integro-differential equations similar to 1 . As a result, only the first $(i-1)$ variables are necessary for the full description of the investigated system. In the case of simple liquid metals, by analogy with the hydrodynamics region, we propose that only three variables, namely local density, local momentum density, and local energy density, are sufficient to reproduce its spectral features at the microscopic level. These variables are implicitly present in $F(k, t), M_{1}(k, t)$ and $M_{2}(k, t)$. From the above reasoning, one can write the following closure $M_{4}(k, t) \approx M_{3}(k, t)$. By means of the Laplace trans- formation of the corresponding equations for $M_{0}(k, t)=$ $F(k, t), M_{1}(k, t), M_{2}(k, t)$ we obtain an expression for the dynamic structure factor in which the static structure factor $S(k)$ and general relaxation parameters of the $i$ th orders $\Omega_{i}^{2}(i=1,2,3,4)$ are contained

$$
\begin{gathered}
S(k, \omega)=\frac{S(k)}{2 \pi} \Omega_{1}^{2} \Omega_{2}^{2} \Omega_{3}^{2}\left(4 \Omega_{4}^{3}-\omega^{2}\right)^{1 / 2}\left\{\Omega_{1}^{4} \Omega_{3}^{4}\right. \\
+\omega^{2}\left(\Omega_{1}^{4} \Omega_{4}^{2}-2 \Omega_{1}^{2} \Omega_{3}^{4}-\Omega_{1}^{4} \Omega_{3}^{2}+2 \Omega_{1}^{2} \Omega_{2}^{2} \Omega_{4}^{2}\right. \\
\left.-\Omega_{1}^{2} \Omega_{2}^{2} \Omega_{3}^{2}+\Omega_{2}^{4} \Omega_{4}^{2}\right)+\omega^{4}\left(\Omega_{3}^{4}-2 \Omega_{1}^{2} \Omega_{4}^{2}\right. \\
\left.\left.+2 \Omega_{1}^{2} \Omega_{3}^{2}-2 \Omega_{2}^{2} \Omega_{4}^{2}+\Omega_{2}^{2} \Omega_{3}^{2}\right)+\omega^{6}\left(\Omega_{4}^{2}-\Omega_{3}^{2}\right)\right\} .
\end{gathered}
$$

Now we use the theory proposed above for the evaluation of $S(k, \omega)$ in liquid cesium near its melting point at $T=308 \mathrm{~K}$. The quantities needed for the calculation are $S(k)$ and $\Omega_{i}^{2}(i=1,2,3,4)$. The numerical static structure data were obtained by Bodensteiner et al. [5], and we use these $S(k)$ data in our calculations. The first two relaxation parameters $\Omega_{1}^{2}$ and $\Omega_{2}^{2}$ are defined as $\Omega_{1}^{2}=K_{B} T k^{2} /[M S(k)], \Omega_{2}^{2}=\omega_{l}^{2}-\Omega_{1}^{2}$, where $\omega_{l}^{2}=$ $3 \Omega_{1}^{2} S(k)+N / M V \int d r g(r)[1-\cos (k r)] \nabla_{z}^{2} u(r)$. Here $K_{B} T$ is the thermal energy; $g(r)$ is the radial distribution function, and $u(r)$ is the pair interparticle interaction potential (axis $z$ is chosen in the direction of the wave vector $\mathbf{k})$. However, to calculate the second frequency parameter $\Omega_{2}^{2}$, we use the known Hubbard-Beeby approximation [8] $\Omega_{2}^{2}=3 \Omega_{1}^{2} S(k)+\omega_{E}^{2}[1-3 \sin (x) / x-$ $\left.6 \cos (x) / x^{2}+6 \sin (x) / x^{3}\right]-\Omega_{1}^{2}$. Here $x=k R_{0}$ with $R_{0}=$ $4.8 \AA$ and the Einstein frequency $\omega_{E}^{2}$ is taken to be $4.12 \mathrm{ps}^{-1}$, which is the value obtained by Bodensteiner [5]. The theoretical formulas for $\Omega_{3}^{2}$ and $\Omega_{4}^{2}$ cannot be used in calculations for one reason. The final result of these calculations has gross errors. Therefore, these parameters can be defined by comparing theoretical and experimental results. Namely, we found the third and the forth relaxation frequency parameters $\left(\Omega_{3}^{2}\right.$ and $\Omega_{4}^{2}$ ) were found by us from two mutually independent conditions: the behavior of $S(k, \omega)$ in the points of a central $(\omega=0)$ and a side $(\omega \neq 0)$ maximums.

We have calculated $S(k, \omega)$ in the low- $k$ region $k=$ $0.4 \sim 0.9 \AA^{-1}$, where high-frequency peaks were experimentally observed. The results for the representative wave vectors are shown in Fig. 1. It is clear in Fig. 1 that our theory describes adequately the collective density excitations in spectra of $S(k, \omega)$ for liquid cesium. For the investigated wave-vector region, we have achieved a good agreement with the experiment [5]. In Fig. 2, we report a set of normalized dynamic structure factors 


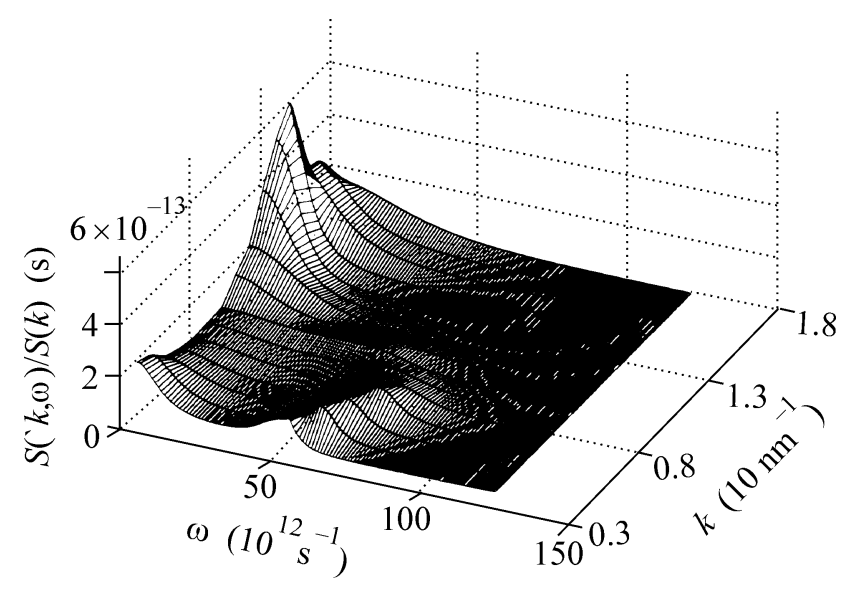

Fig. 2. Dynamic structure factor calculated on the basis theory for liquid cesium at $308 \mathrm{~K}$

$S(k, \omega) / S(k)$ as calculated from our theory for the wave vector region $0.4 \leq k \leq 1.8 \AA^{-1}$. In this figure, one can see certain changes in the position and altitude of the central and side peaks at different values of the wave vector $\mathbf{k}$. As can be seen in Fig. 2, side peaks persist up to $k \sim 1.1 \AA^{-1}$, i.e., very close to the position of the first peak in the static structure factor $\left(k \sim 1.4 \AA^{-1}\right)$. This again shows that the liquid supports collective excitations for wavelengths comparable with the mean interparticle spacing, a distinctive characteristic of liquid metals [6].

To understand the nature of the collective excitations below $k=1 \AA^{-1}$, we have studied the behavior of the frequency-dependent non-Markovity parameter $\varepsilon_{1}(k, \omega)$. This parameter was at first entered [9] on the basis of parameter $\varepsilon_{0}=\tau_{0} / \tau_{1}$, where the relaxation times $\tau_{0}$ and $\tau_{1}$ are calculated by Eq. 3 . By this non-Markovity parameter [10], all relaxation processes can be divided into Markovian scenario, when $\varepsilon_{0} \longrightarrow \infty$, quasi-Markovian scenario at $\varepsilon_{0}>1$, and non-Markovian scenario at $\varepsilon_{0} \sim 1$ The generalized parameter $\varepsilon_{1}(k, \omega)$ is defined at fixed $\mathrm{k}$ by the expression

$$
\begin{gathered}
\varepsilon_{j}(\omega)=\left[\frac{\mu_{j-1}(\omega)}{\mu_{j}(\omega)}\right]^{1 / 2}, \\
\mu_{j}(\omega)=\left\{\operatorname{Re}\left[\int d t M_{j} e^{i \omega t}\right]\right\}^{2} .
\end{gathered}
$$

Here $\mu_{j}(\omega)$ is the power spectra of the $i$ th relaxation level was introduced. In Fig. 3 we present the results of calculations for the frequency-dependent non-Markovity parameter $\varepsilon_{1}(k, \omega)$ at several values of wave vectors $k$. It can be seen in Fig. 3 that the values of $\varepsilon_{1}(k, \omega)$ show the alternation of maxima and minima. In addition, $\varepsilon_{1}(k, \omega)$ and $S(k, \omega)$ maxima in the low- $k$ region are located on approximately the same frequencies that are associated with collective excitations in liquid metal.

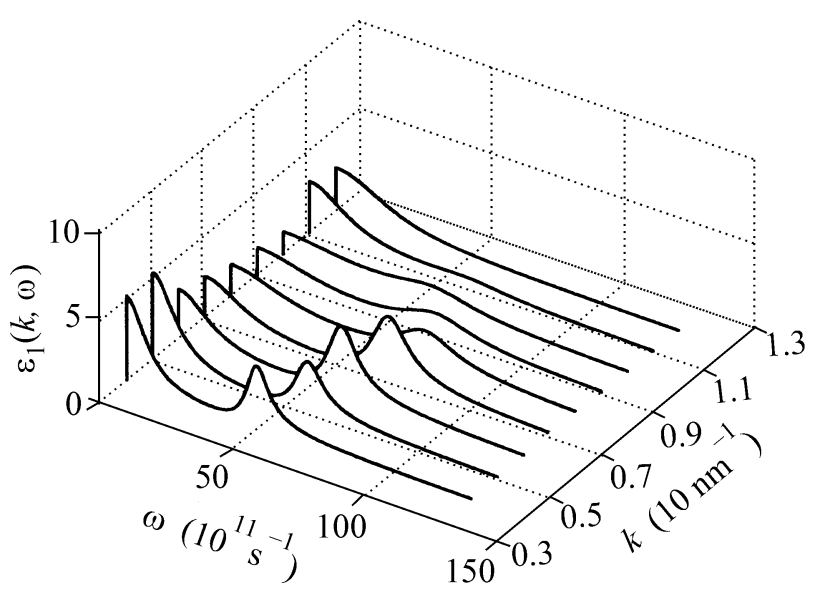

Fig. 3. Frequency dependence of non-Markovity parameter $\varepsilon_{1}(k, \omega)$ for $4<k<12 \mathrm{~nm}^{-1}$

The amplitudes of these peaks quickly fall down as $k$ increases. This is due to the amplification of non-Markovity effects. The occurrence of $\varepsilon_{1}(k, \omega)$ peaks in the region of collective excitations indicates an essential amplification of quasi-Markovity on propagation of ion density fluctuations. High-frequency collective excitations disappear smoothly with increasing $k$, simultaneously non-Markovity is further enhanced. Similarly, an increase in $\varepsilon_{1}(k, \omega=0)$ means an enhancement of randomness in the thermal motion of particles. Thus, the observed frequency behavior of the parameter $\varepsilon_{1}(k, \omega)$ consists in consecutive alternation of quasiMarkovian and non-Markovian relaxation scenarios of behavior. The similar alternation cannot be received within the framework of any other theory.

In this Letter, we have presented the theory, based on Zwanzig-Mori's MF formalism [7] and the idea of TSI, which allows one to describe the dynamic structure spectra in all experimentally investigated region of the wave vector. The long-range memory and the short time correlations have affected the analysis of nonMarkov properties of the collective dynamics in liquid cesium. In this way we are going to establish the nonMarkov nature of collective excitations in liquid cesium at the low- $k$ values.

This work was partially supported by Russian Foundation of Basic Researches (Grant \#02-02-16146). The authors acknowledge Prof. A. G. Novikov and Dr. T. Scopigno for stimulating discussions of scattering data and Dr. L. O. Svirina for technical assistance.

\section{REFERENCES}

1. J. R. D. Copley and M. Rowe, Phys. Rev. A 9, 1656 (1974); O. Söderstrem, Phys. Rev. A 23, 785 (1981); O. Söderstrem, J. R. D. Copley, J. B. Suck, et al., J. Phys. F 10, L151 (1980); U. Dahlborg and K. E. Larsson, Ark. Fys. 33, 271 (1966); C. Morkel and W. Gläser, Phys. Rev. A 33, 3383 (1986); A. Stangl, C. Morkel, U. Balu- 
cani, et al., J. Non-Cryst. Solids 205-207, 402 (1996); A. G. Novikov, V. V. Savostin, A. L. Shimkevich, et al., Physica B (Amsterdam) 228, 312 (1996).

2. S. Kambayashi and G. Kahl, Phys. Rev. A 46, 3255 (1992).

3. M. Canales, L. E. González, and Á. Padro, Phys. Rev. E 50, 3656 (1994); U. Balucani, A. Torcini, and R. Vallauri, Phys. Rev. B 47, 3011 (1993).

4. F. Yoshida, J. Phys. F 8, 411 (1978); F. Yoshida and S. Takeno, J. Phys. C 11, 2895 (1978); R. K. Sharma and K. Tankeshwar, Phys. Rev. E 55, 564 (1997).
5. T. Bodensteiner, Chr. Morkel, and B. Dorner, Phys. Rev. A 45, 5709 (1992).

6. U. Balucani and M. Zoppi, Dynamics of the Liquid State (Clarendon, Oxford, 1994).

7. R. Zwanzig, Phys. Rev. 124, 1338 (1961); H. Mori, Prog. Theor. Phys. 33, 423 (1965).

8. J. Hubbard and J. L. Beeby, J. Phys. C 2, 556 (1969).

9. R. M. Yulmetyev, P. Hänggi, and F. Gafarov, Phys. Rev. E 62, 6178 (2000).

10. V. Yu. Shurygin, R. M. Yulmetyev, and V. V. Vorobjev, Phys. Lett. A 148, 199 (1990).

SPELL: OK 